ISSN 2219-6722 / ISSNE 2222-2707

\title{
DIVERSIFICACIÓN DE LAS EXPORTACIONES COMO ESTRATEGIA PARA EL CRECIMIENTO ECONÓMICO DE HONDURAS
}

\author{
José Bayardo Cabrera Rosales \\ Universidad Nacional Autónoma de Honduras (UNAH) \\ Facultad de Ciencias Economicas, Administrativa y Contables (FCEAC) \\ Instituto de Investigaciones Económicas y Sociales (IIES) \\ Ciudad universitaria, edificio C2, primer piso, Tel/fax: + (504) 2216-6100 Ext. 100894 \\ jose.cabrera@unah.edu.hn
}

\section{RESUMEN}

La siguiente investigación tiene como objetivo principal evaluar la diversificación de las exportaciones como variable determinante del crecimiento económico, así como evaluar si una concentración de las exportaciones de productos hondureños tiene un efecto negativo para el crecimiento de las exportaciones, el documento está dividido en seis secciones; la primera de ellas expone una introducción de la temática abordada dentro de la investigación, la segunda sección contiene el estado del arte así como antecedentes empíricos que aportan conocimiento para abordar el tema, la sección número tres presenta la metodología empleada para el desarrollo de la investigación, la sección cuatro expone los resultados encontrados en el desarrollo de la investigación, y en la sección número cinco se presentan las conclusiones y algunas recomendaciones.

Palabras Clave: Concentración, Diversificación, Exportaciones, Crecimiento Económico. 


\title{
DIVERSIFICATION OF EXPORTS AS A STRATEGY FOR THE ECONOMIC GROWTH OF HONDURAS
}

\author{
José Bayardo Cabrera Rosales \\ Universidad Nacional Autónoma de Honduras (UNAH) \\ Facultad de Ciencias Economicas, Administrativa y Contables (FCEAC) \\ Instituto de Investigaciones Económicas y Sociales (IIES) \\ Ciudad universitaria, edificio C2, primer piso, Tel/fax: + (504) 2216-6100 Ext. 100894 \\ jose.cabrera@unah.edu.hn
}

\begin{abstract}
The following research has as main objective to evaluate the diversification of exports as a key variable of economic growth, as well as assessing whether a concentration of exports of Honduran products chas a negative effect on the growth of exports, the document is divided into six sections ; the first one presents an overview of the topics addressed in the investigation, the second section contains the state of the art and empirical background that provide knowledge to address the issue, the number Section three presents the methodology for the development of research, section four presents the results found in the research development, and the number five section some conclusions and recommendations.
\end{abstract}

Keywords: Concentration, Diversification, Export, Economic Growth 


\section{INTRODUCCIÓN}

En el año 2015 el producto Interno Bruto (PIB) de Honduras a precios contantes fue de 189,771 millones de Lempiras, lo cual representó un crecimiento económico de $3.6 \%$ respecto al año 2014, del total del PIB hondureño en 2015, 12.67\% fue contribución por parte del sector agrícola, $13.9 \%$ fue por parte del comercio, y el sector con más aporte al PIB fue la industria manufacturera con $16.8 \%$.

Históricamente Honduras ha sido un país que exporta principalmente productos agrícolas, tal es así como por mucho tiempo se le reconoció como el principal exportador de banano a nivel mundial, actualmente se ha convertido en uno de los principales exportadores de café a nivel mundial. En el año 2015 Honduras exportó 9,145 millones de US\$, no obstante, importó 12,891 millones de US\$, lo cual dejó una balanza comercial de US\$ $-3,746$ millones, del total de las exportaciones de Honduras en $2015,10.8 \%$ fueron de café y $5.5 \%$ fueron de banano.

Si bien existen diversas teorías que argumentan que concentrar las exportaciones, es decir, especializarse en un producto en específico puede generar beneficios ya que así se abarata costos y se puede producir ese producto de manera más eficaz, no obstante, existe el costo de oportunidad que se pierde al dejar de impulsar otros productos que podría traer mayores ganancias, tal es así que Honduras al estar tan concentrado en el café, cuando se desató el fenómeno de la roya, sus exportaciones se vieron mermadas, también el café es un producto de precio muy fluctuante y además Honduras no genera valor agregado por lo cual es un producto eminentemente agrícola.

\section{OBJETIVOS}

\subsection{Objetivo General}

Describir los principales bienes de exportación de
Honduras, midiendo el índice de concentración de Herfindal Hirchman (IHH) para observar el comportamiento de las exportaciones de Honduras, en el periodo de tiempo de 1980 a 2015.

\subsection{Objetivos Específicos}

-Identificar y describir el comportamiento del principal producto de exportación de Honduras.

-Medir el IHH en las exportaciones de Honduras durante el periodo 1980-2015

-Dar una revisión a la literatura existente de la relación entre la diversificación de las exportaciones y el crecimiento económico

\section{ESTADO DEL ARTE}

Para entender el comercio exterior entre naciones se debe remontar hasta la teoría planteada por David Ricardo, en la cual se menciona que los países comercian entre si principalmente porque existe dotación de factores de producción y porque además buscan economías a escala; es decir, una mayor producción a un menor costo, también en la teoría se expone el concepto de ventaja absoluta y ventaja comparativa.

Por otro lado, la ventaja comparativa se refiere a la ventaja que tiene un país en la en el costo de producción respecto a otro país, es decir, un país tendrá ventaja absoluta respecto a otro país si su costo de trabajo es menor que al otro país. En cambio, el concepto de ventaja comparativa, Ricardo planteó que es la ventaja que un país posee en la producción de un bien en función de otro bien, es decir, el costo de dejar de producir el bien "A" por producir el bien "B", en ese caso se dice que cuando un país renuncia a menos unidades del bien "B" para producir "A", así un país obtiene ventaja comparativa respecto a otros. (Krugman, Obstfeld, Garuz y Díaz, 2001). 
Dentro de las conclusiones de Ricardo están que el comercio exterior amplía la posibilidad de producción de una economía, los salarios de los países socios comerciales mejoran y, además, la especialización en la producción de un solo bien para un país es de vital importancia ya que esa es la manera de ser más competitivo respecto a los demás competidores. Es importante mencionar que el modelo cuenta con los siguientes supuestos: (1) existen solo dos bienes para comerciar; (2) solo hay un factor productivo (trabajo); (3) existe solo dos países que comercian entre sí; (4) el factor de trabajo se mueve libremente en la producción de ambos bienes y (5) existe competencia perfecta. La rigurosidad de estos supuestos y la simplicidad del modelo es lo que atrajo muchas críticas al modelo Ricardiano. (Krugman, Obstfeld, Garuz y Díaz, 2001).

Por otro lado,Heckscher-Ohlin plantean también su modelo del comercio exterior, a diferencia del modelo Ricardiano, el modelo Heckscher-Ohlin (HO) establece otros supuestos: i) existen dos naciones, ii) ambos países utilizan la misma tecnología para producir, iii) existe dos factores de producción; trabajo capital, iv) existe dos bienes de producción, v) existe competencia perfecta, vi) las preferencias son iguales en ambas naciones, vii) existe movilidad perfecta de factores de producción, viii) no existe costo de transporte, ix) se emplea todos los recursos disponibles, $\mathrm{y}$ x) existe un comercio equilibrado entre ambas naciones.

La principal diferencia con el modelo propuesto por David Ricardo radica en que HeckscherOhlin establece la existencia de dos bienes que se comercian. La principal conclusión a la que llega el modelo de $\mathrm{H}-\mathrm{O}$ es que los países exportaran el bien en el cual el factor de producción para fabricarlo sea abundante en el país, e importará el bien el cual se le haga más dificultoso fabricarlo debido a que escasea su factor de producción en el ámbito local. (Krugman, Obstfeld, Garuz y Díaz, 2001)

\section{1.-Antecedentes}

Sannassee, Seetanah, y Lamport, (s.f.), elaboran un estudio para el caso del país de Mauricio en el cual analizaron la relación existente de la diversificación de las exportaciones de dicho país y el crecimiento económico, los autores concluyen que efectivamente existe relación positiva entre la diversificación de las exportaciones y el crecimiento económico de Mauricio, no obstante, a corto plazo el proceso de diversificación de las exportaciones para un país en desarrollo resulta ser muy costoso, por lo cual el papel que juega el gobierno es fundamental debido a que se debe crear el ambiente propicio y crear las políticas públicas para incentivar los sectores que se consideran competitivos o con alto potencial de exportación para un país.

Agosín (2009), analizó el crecimiento y la diversificación de las exportaciones en las economías emergentes, Agosín observó la cantidad de productos exportados por algunos los países asiáticos y otros países seleccionados de Latinoamérica, con lo cual su hipótesis que quiso probar fue que una diversificación de la cartera de exportaciones conllevaba un incremento en el crecimiento económico de los países, a través de la estimación de un modelo econométrico Agosín descubrió y concluye que la diversificación de las exportaciones contribuyó de manera considerable en el periodo 1980-2008 al crecimiento económico de Latinoamérica y Asia.

Para el caso de Centroamérica visto como el Mercado Común Centro Americano (MCCA), Castillo, Aguilera y García (2009) analizaron la diversificación de las exportaciones del MCCA, ellos estudiaron la diversificación de los mercados y no de los productos de exportación, evaluando que Estados Unidos constituye un mercado al cual el MCCA exporta un total de 32\% de sus productos, para el cálculo de la diversificación 
ISSN 2219-6722 / ISSNE 2222-2707

utilizaron el índice Herfindahl Hirschman (IHH), además de modelos econométricos los cuales buscaban determinar la importancia de la diversificación de mercados de exportación, los resultados obtenidos Castillo, Aguilera y García, fueron que una diversificación es deriva un mayor beneficio para Centroamérica, no obstante, que la crisis económica de finales de la década pasada hicieron mella al estar tan concretadas las exportaciones hacia un solo país (Estados Unidos).

\section{2.- Î́ndice Herfindahl Hirschman}

El índice Herfindahl Hirchman es muy utilizado para medir la concentración de los mercados, generalmente a través del IHH se puede argumentar si un mercado es competitivo o si está siendo monopolizado, no obstante, el IHH puede ser empleado para un sinfín de tareas que pueden ir desde medir la diversificación de cartera de un inversor, hasta el caso de la presente investigación, medir la diversificación o concentración de las exportaciones de una región Kwoka (1985). Su método de cálculo es el siguiente:

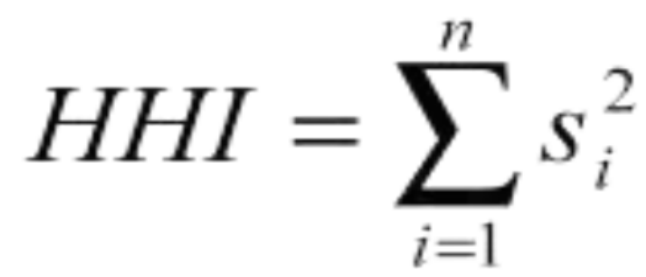

Es decir, la suma de los cuadrados de la participación de cada variable dentro del total, los resultados del IHH se pueden interpretar de la siguiente manera:(ver tabla No.1)

\begin{tabular}{|l|l|}
\hline Resultado & Interpretación \\
\hline $1801-10000$ & Altamente concentrado \\
\hline $1000-1800$ & Moderadamente concentrado \\
\hline $1-999$ & No concentrado \\
\hline
\end{tabular}

Tabla $N^{\circ} 1$ Interpretación de Resultados IHH

Fuente: Elaboración propia

\section{METODOLOGÍA DE LA INVESTIGACIÓN}

La investigación es de corte descriptivo explicativo, ya que describe el comportamiento de la economía hondureña del periodo 1980 al 2015 así como el comportamiento de las exportaciones. Además, se pretende explicar la relación existente entre la diversificación de las exportaciones y el crecimiento económico.

Para medir el grado de diversificación de las exportaciones se calculó el IHH a partir del dato de exportaciones que proporciona el Banco Central de Honduras $(\mathrm{BCH})$, asimismo el crecimiento económico que se utilizó es el proporcionado por el $\mathrm{BCH}$, es importante mencionar que no se toma en cuenta productos de la maquila.

Para medir la relación existente entre la diversificación de exportaciones y el crecimiento económico, se utilizó el coeficiente de correlación de Pearson, el cual se calculó utilizando el software estadístico STATA 12. Una Vez teniendo el resultado de los cálculos se procedió a realizar conjeturas respecto a los mismos, estos se explican en la sección 5 del documento.

\section{RESULTADOS}

La economía hondureña se considera una Revista Economía y Administración (E\&A) / V0L. 835 
economía pequeña y muy abierta al comercio internacional, en el año 2015 el coeficiente de apertura fue de 107.8, es decir muy abierta, es de resaltar que este coeficiente alto ha venido siendo así desde mediados de la década de los 90's. Las exportaciones de Honduras en el año 2015 ascendieron a 9,144 Millones de US\$ en términos nominales, en términos reales fueron de 3,897 millones de US\$ lo que significó un incremento de $7 \%$ respecto al año 2014 , cabe resaltar que los años previos debido al fenómeno de la roya y a una disminución del precio internacional del café, las exportaciones hondureñas habían venido a la baja en los años 2014 y 2013, en cuanto al crecimiento de la economía hondureña en el año 2015 fue de $3.6 \%$, lo cual es el promedio de crecimiento en Honduras a partir del año 2010.

\section{1.- Principales productos de exportación de Honduras}

El principal producto de exportación de Honduras en el año 2015 fue el café, el cual generó 986 millones de US\$ en materia de divisas $(25,8 \%)$, si bien en el 2015 este producto fue el mayor generador de divisas para Honduras, el café se ha enfrentado fenómenos que han mermado su exportación en los últimos tres años, previo a la roya el café en el año 2012 generó 1402 millones de US\$ en divisas para Honduras, lo cual es de resaltar su vulnerabilidad ante fenómenos climáticos y también su alta dependencia del precio internacional, no obstante, es importante mencionar que Honduras es un destacado exportador de café a nivel mundial ubicándose en el sexto lugar de exportaciones de café.

El segundo producto en importancia de exportaciones en 2015 de Honduras fue el banano el cual representó $(13.2 \%)$ del total de exportaciones, el banano históricamente ha sido un producto de exportación importante para Honduras, tal es así como hasta el año 1993 este fue el producto líder de Honduras. El aceite de palma toma relevancia en Honduras a partir del año 2000, hasta ubicarse como el tercer producto en importancia para Honduras aportando (6.3\%) de las exportaciones en el 2015. Si agregamos el camarón cultivado con $(4.7 \%)$ es de resaltar que entre los cuatro principales productos representan casi el $(50 \%)$ de las exportaciones hondureñas (ver figura No. 1), lo cual deja a entrever la poca diversidad que cuenta Honduras en sus bienes de exportación.

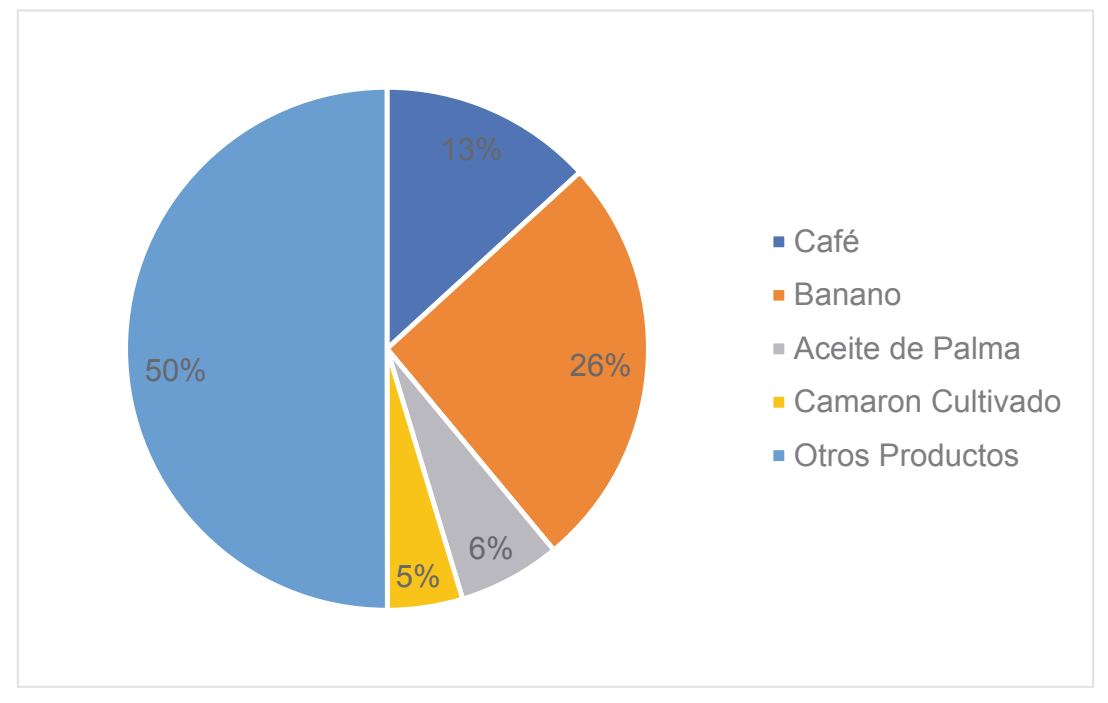

Figura No.1 Principales exportaciones de Honduras año 2015

Fuente: Elaboración propia con datos del Banco Central de Honduras 
ISSN 2219-6722 / ISSNE 2222-2707

\section{2.- Diversificación de las exportaciones y crecimiento económico}

El IHH de Honduras para el año 2015 fue de 1486 (Ver anexo No. 1) lo cual significa que las exportaciones hondureñas están concentradas moderadamente. Históricamente las exportaciones han sido concentradas con IHH arriba de 2000, no obstante, con el auge de la producción de palma africana, así como el impulso del plástico y cartón, tilapias entre otros, la diversidad de productos de exportación aumentó moderadamente y conllevo a obtener un IHH en promedio de 1500 a partir del año 2000.

Considerando el IHH calculado para las exportaciones de Honduras, se prosiguió a elaborar un índice de correlación de Pearson con el fin de determinar si la diversificación de las exportaciones tiene efecto en dos aspectos:

La primera hipótesis es saber si a mayor diversificación de las exportaciones existe un incremento en las exportaciones, es decir, si la concentración o especialización tiene efecto positivo o negativo en el crecimiento de las mismas, para elaborar el cálculo se utilizó el cálculo del IHH a partir del año 1980 hasta el año 2015 con datos del $\mathrm{BCH}$, y para el crecimiento de las exportaciones se utilizó la variación de las exportaciones reales de Honduras del año 1980 hasta el 2015.

Utilizando el software STATA 12 se obtuvo coeficiente de correlación de -0.2475 , el signo negativo se interpreta como que existe una relación inversa entre el crecimiento de las exportaciones y la concentración de las mismas, es decir, se espera que si Honduras concentra las exportaciones, estas tienden a disminuir o a crecer con menor intensidad, no obstante, el valor de 0.24 obtenido se interpreta como que la correlación obtenida es débil, ya que para ser considerada esta correlación debería resultar superior al menos a 0.8 .

La segunda hipótesis consiste en que a mayor diversificación existe un mayor crecimiento económico, el resultado calculado fue de -0.2399 , nuevamente obteniendo un signo negativo, lo cual significa que el crecimiento económico y la concentración de las exportaciones están inversamente relacionados, sin embargo, es importante mencionar que, al obtener un valor bajo, ambos resultados no son contundentes, los resultados obtenidos se visualizan en la figura No. 2

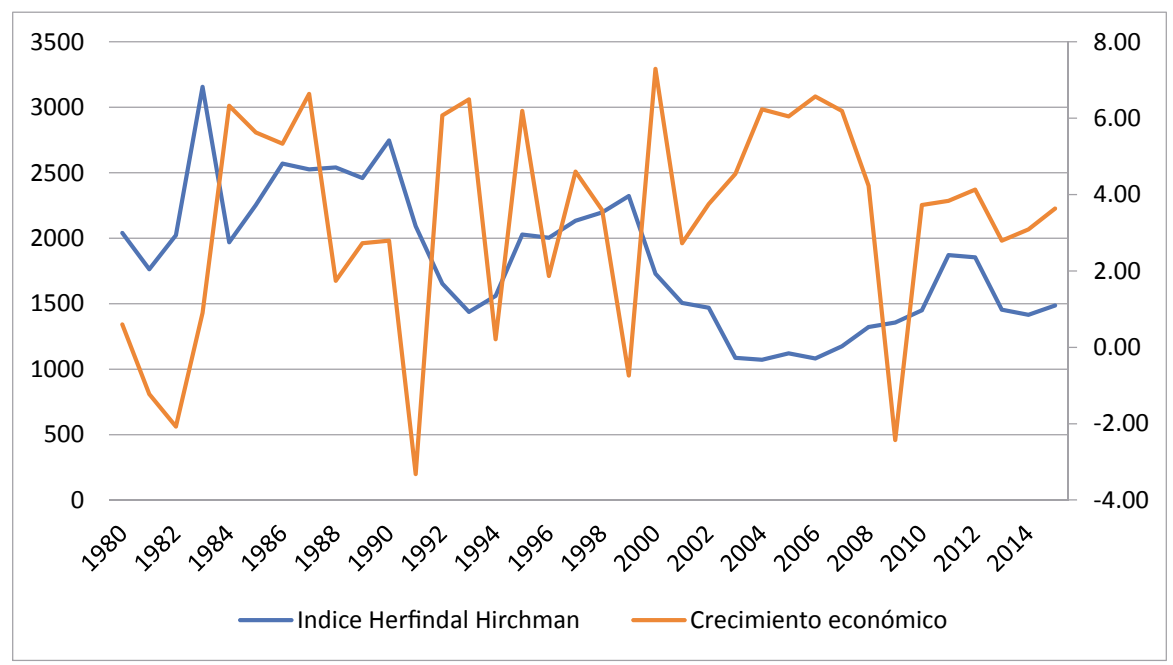

Figura No.2 IHH y crecimiento económico de Honduras 1980-2015

Fuente: Elaboración propia con datos del $\mathrm{BCH}$. 


\section{CONCLUSIONES Y RECOMENDACIONES}

Si bien el resultado de correlación de la diversificación de exportaciones y el crecimiento económico en Honduras resultó ser tal y como la teoría lo plantea, es decir, que cuando un país cuenta con mayor diversidad de productos de exportación esto repercutirá en su crecimiento económico positivamente, en los resultados obtenidos se observó un bajo coeficiente de correlación por lo cual, lo más acertado y lo recomendado para futuros adelantos dentro de esta investigación sería realizar un modelo econométrico, con la intención de considerar otras variables que además de la diversificación de las exportaciones estén estrechamente relacionadas con el crecimiento de la economía.

Es importante mencionar que la especialización en la exportación de café para Honduras ya le ha traído problemas, tanto así que, con la caída del precio del café y el fenómeno de la roya, las exportaciones hondureñas disminuyeron $11.8 \%$ del 2012 al 2013.

Teniendo en cuanta lo expuesto anteriormente, pareciera que lo conveniente para economías dependientes de un solo producto como Honduras sería orientar e impulsar mercados emergentes para evitar eventos como los del café, no obstante, esto debe realizarse con suma precaución y se debería evaluar los productos en los cuales Honduras podría ser más competitivo respecto a sus principales socios comerciales, es decir el MCC.

\section{REFERENCIAS}

Agosin, M. (2009). Crecimiento y diversificación de exportaciones en economías emergentes. Disponible en http://www. cepal.org/publica pdf

Banco Central de Honduras (2016). Estadísticas Macroeconómicas. Disponible en www. bch.com

Castillo, J., Aguilera, E., y García C. (2013). Centroamérica: lo impostergable de una diversificación comercial coherente. Problemas del desarrollo, 44(174), 119-138. Disponible en $\quad$ http://www.scielo.org.mx/scielo. php? script $=$ sci arttext\&pid $=$ S0301$\underline{70362013000300006 \& \operatorname{lng}=\mathrm{es} \& \operatorname{tlng}=\mathrm{es}}$.

Kwoka Jr, J. E. (1985). Herfindahl Index in Theory and Practice, The. Antitrust Bull., 30, 915.

Krugman, P., Obstfeld, M., Garuz, J. T., Diaz, E. S., \& Taña, J. O. (2001). Economía internacional. Pearson Education.

Sannassee, R., Seetanah, B. y Lamport, M. (s.f.) Diversificación de las exportaciones y crecimiento económico: el caso de Mauricio. Disponible en https://www. wto.org/spanish/res_s/booksp_s/cmark chap1_s.pdf 


\section{ANEXOS}

\section{Anexo No. 1}

Exportaciones Hondureñas 1980-1988 (valores corrientes en millones de Dólares US\$)

\begin{tabular}{|c|c|c|c|c|c|c|c|c|c|}
\hline & 1980 & 1981 & 1982 & 1983 & 1984 & 1985 & 1986 & 1987 & 1988 \\
\hline BANANO & 456.00 & 426.60 & 436.60 & 40.30 & 456.80 & 547.00 & 513.50 & 648.50 & 356.40 \\
\hline CAFÉ & 408.20 & 345.70 & 306.20 & 302.40 & 337.90 & 370.00 & 644.10 & 416.70 & 192.10 \\
\hline MADERA & 72.40 & 86.30 & 89.30 & 30.80 & 67.40 & 68.20 & 64.60 & 72.60 & 29.80 \\
\hline Carne refrigerada & 121.50 & 92.90 & 67.80 & 62.70 & 49.20 & 36.30 & 39.30 & 42.20 & 20.30 \\
\hline ACEÍTE DE PALMA & 0.00 & 0.00 & 0.00 & 0.00 & 0.00 & 0.00 & 0.00 & 0.00 & 0.00 \\
\hline PLATA & 0.00 & 31.50 & 18.60 & 35.10 & 40.50 & 26.00 & 25.10 & 10.80 & 9.90 \\
\hline PLOMO & 0.00 & 16.80 & 8.50 & 7.70 & 15.90 & 11.90 & 12.30 & 3.70 & 7.30 \\
\hline ZINC & 0.00 & 24.50 & 23.90 & 33.10 & 69.80 & 59.90 & 52.50 & 23.30 & 29.80 \\
\hline AZÚCAR & 58.70 & 93.10 & 43.20 & 55.70 & 50.60 & 42.90 & 25.00 & 39.00 & 14.40 \\
\hline CAMARÓN CULTIVADO & 0.00 & 0.00 & 0.00 & 0.00 & 0.00 & 0.00 & 0.00 & 0.00 & 0.00 \\
\hline CAMARÓN DE EXTRACCIÓN & 0.00 & 0.00 & 0.00 & 0.00 & 0.00 & 0.00 & 0.00 & 0.00 & 0.00 \\
\hline Camaron & 0.00 & 0.00 & 0.00 & 0.00 & 0.00 & 0.00 & 0.00 & 0.00 & 49.40 \\
\hline Camarones y Langostas & 46.80 & 52.50 & 55.90 & 72.00 & 77.80 & 81.90 & 90.70 & 122.80 & 0.00 \\
\hline LANGOSTAS & 0.00 & 0.00 & 0.00 & 0.00 & 0.00 & 0.00 & 0.00 & 0.00 & 32.60 \\
\hline TILAPIAS & 0.00 & 0.00 & 0.00 & 0.00 & 0.00 & 0.00 & 0.00 & 0.00 & 0.00 \\
\hline MELONES Y SANDÍAS & 0.00 & 0.00 & 0.00 & 0.00 & 0.00 & 0.00 & 0.00 & 0.00 & 0.00 \\
\hline PIÑAS & 0.00 & 0.00 & 0.00 & 0.00 & 0.00 & 0.00 & 0.00 & 0.00 & 0.00 \\
\hline LEGUMBRES Y HORTALIZAS & 0.00 & 0.00 & 0.00 & 0.00 & 0.00 & 0.00 & 0.00 & 0.00 & 0.00 \\
\hline PREPARACIONES LEGUMBRES Y FRUTAS & 0.00 & 0.00 & 0.00 & 0.00 & 0.00 & 0.00 & 0.00 & 0.00 & 0.00 \\
\hline PUROS O CIGARROS & 0.00 & 0.00 & 0.00 & 0.00 & 0.00 & 0.00 & 0.00 & 0.00 & 0.00 \\
\hline CIGARRILLOS & 0.00 & 0.00 & 0.00 & 0.00 & 0.00 & 0.00 & 0.00 & 0.00 & 0.00 \\
\hline TABACO & 27.40 & 26.70 & 21.50 & 21.60 & 21.00 & 17.30 & 10.60 & 7.10 & 3.10 \\
\hline Pure y pasta de Frutas & 0.00 & 0.00 & 0.00 & 0.00 & 0.00 & 0.00 & 0.00 & 0.00 & 0.00 \\
\hline MANUFACTURAS DE MADERA & 0.00 & 0.00 & 0.00 & 0.00 & 0.00 & 0.00 & 0.00 & 0.00 & 0.00 \\
\hline MUEBLES DE MADERA & 0.00 & 0.00 & 0.00 & 0.00 & 0.00 & 0.00 & 0.00 & 0.00 & 0.00 \\
\hline TEXTILES & 0.00 & 0.00 & 0.00 & 0.00 & 0.00 & 0.00 & 0.00 & 0.00 & 0.00 \\
\hline JABONES Y DETERGENTES & 41.00 & 25.90 & 18.60 & 0.00 & 0.00 & 0.00 & 0.00 & 0.00 & 0.00 \\
\hline PLÁSTICOS Y SUS MANUFACTURAS & 0.00 & 0.00 & 0.00 & 0.00 & 0.00 & 0.00 & 0.00 & 0.00 & 0.00 \\
\hline Algodón & 26.90 & 24.90 & 13.00 & 0.00 & 0.00 & 0.00 & 0.00 & 0.00 & 0.00 \\
\hline PAPEL Y CARTÓN & 0.00 & 0.00 & 0.00 & 0.00 & 0.00 & 0.00 & 0.00 & 0.00 & 0.00 \\
\hline Otros Productos & 385.30 & 259.80 & 204.30 & 673.30 & 284.70 & 267.80 & 230.80 & 264.70 & 93.90 \\
\hline TOTAL BIENES & 1644.20 & 1507.20 & 1307.40 & 1334.70 & 1471.60 & 1529.20 & 1708.50 & 1651.40 & 839.00 \\
\hline
\end{tabular}

Fuente: Elaboración propia con datos del $\mathrm{BCH}$. 
Anexo No. 2

Exportaciones Hondureñas 1989-1998 (valores corrientes en millones de Dólares US\$)

\begin{tabular}{|c|c|c|c|c|c|c|c|c|c|c|}
\hline & 1989 & 1990 & 1991 & 1992 & 1993 & 1994 & 1995 & 1996 & 1997 & 1998 \\
\hline BANANO & 351.70 & 366.30 & 314.44 & 256.14 & 225.60 & 155.10 & 214.20 & 279.80 & 212.00 & 219.60 \\
\hline CAFÉ & 190.90 & 180.90 & 145.97 & 147.68 & 124.60 & 200.10 & 349.30 & 278.90 & 326.30 & 429.80 \\
\hline MADERA & 25.40 & 16.10 & 15.31 & 15.60 & 21.90 & 21.30 & 19.00 & 21.80 & 19.50 & 17.10 \\
\hline Carne refrigerada & 19.80 & 24.80 & 31.42 & 37.07 & 39.30 & 39.00 & 13.00 & 11.00 & 11.00 & 4.00 \\
\hline ACEÍTE DE PALMA & 0.00 & 0.00 & 0.00 & 0.00 & 0.00 & 0.00 & 0.00 & 0.00 & 0.00 & 0.00 \\
\hline PLATA & 8.10 & 4.00 & 4.27 & 5.16 & 3.50 & 4.40 & 5.50 & 4.70 & 6.50 & 7.80 \\
\hline PLOMO & 6.00 & 3.60 & 3.31 & 7.93 & 1.80 & 1.70 & 1.80 & 2.70 & 4.60 & 3.20 \\
\hline ZINC & 60.50 & 34.30 & 36.40 & 35.75 & 23.00 & 21.10 & 27.10 & 25.70 & 53.80 & 36.30 \\
\hline AZÚCAR & 10.20 & 11.40 & 8.42 & 5.37 & 5.20 & 4.80 & 6.80 & 9.50 & 12.10 & 10.20 \\
\hline CAMARÓN CULTIVADO & 0.00 & 0.00 & 17.53 & 46.12 & 62.36 & 106.70 & 93.90 & 124.60 & 130.80 & 135.90 \\
\hline CAMARÓN DE EXTRACCIÓN & 0.00 & 0.00 & 36.70 & 22.84 & 32.84 & 26.90 & 30.60 & 18.60 & 20.10 & 21.00 \\
\hline Camaron & 40.00 & 45.90 & 0.00 & 0.00 & 0.00 & & 0.00 & 0.00 & 0.00 & 0.00 \\
\hline Camarones y Langostas & 0.00 & 0.00 & 0.00 & 0.00 & 0.00 & 0.00 & 0.00 & 0.00 & 0.00 & 0.00 \\
\hline LANGOSTAS & 32.40 & 26.90 & 38.71 & 34.13 & 26.80 & 31.90 & 34.10 & 35.00 & 28.20 & 31.80 \\
\hline TILAPIAS & 0.00 & 0.00 & 0.00 & 0.00 & 0.00 & 0.00 & 0.00 & 0.00 & 0.00 & 0.00 \\
\hline MELONES Y SANDÍAS & 0.00 & 0.00 & 12.80 & 10.80 & 23.40 & 24.20 & 25.40 & 30.90 & 39.30 & 43.80 \\
\hline PIÑAS & 0.00 & 0.00 & 12.84 & 20.14 & 21.75 & 19.70 & 21.50 & 22.90 & 18.40 & 18.50 \\
\hline LEGUMBRES Y HORTALIZAS & 0.00 & 0.00 & 0.00 & 0.00 & 0.00 & 0.00 & 0.00 & 0.00 & 0.00 & 0.00 \\
\hline PREPARACIONES LEGUMBRES Y FRUTAS & 0.00 & 0.00 & 0.00 & 0.00 & 0.00 & 0.00 & 0.00 & 0.00 & 0.00 & 0.00 \\
\hline PUROS O CIGARROS & 0.00 & 0.00 & 9.33 & 8.29 & 8.95 & 0.00 & 0.00 & 0.00 & 0.00 & 0.00 \\
\hline CIGARRILLOS & 0.00 & 0.00 & 0.00 & 0.00 & 0.00 & 0.00 & 0.00 & 0.00 & 0.00 & 0.00 \\
\hline TABACO & 3.80 & 1.90 & 0.00 & 0.00 & 6.40 & 5.80 & 5.50 & 5.30 & 7.30 & 8.60 \\
\hline Pure y pasta de Frutas & 0.00 & 0.00 & 6.77 & 4.67 & 4.37 & 0.00 & 0.00 & 0.00 & 0.00 & 0.00 \\
\hline MANUFACTURAS DE MADERA & 0.00 & 0.00 & 10.96 & 14.02 & 15.60 & 0.00 & 0.00 & 0.00 & 0.00 & 0.00 \\
\hline MUEBLES DE MADERA & 0.00 & 0.00 & 0.00 & 0.00 & 0.00 & 0.00 & 0.00 & 0.00 & 0.00 & 0.00 \\
\hline TEXTILES & 0.00 & 0.00 & 14.60 & 18.70 & 21.68 & 0.00 & 0.00 & 0.00 & 0.00 & 0.00 \\
\hline JABONES Y DETERGENTES & 0.00 & 0.00 & 5.04 & 7.74 & 8.93 & 10.40 & 26.30 & 34.10 & 36.90 & 37.20 \\
\hline PLÁSTICOS Y SUS MANUFACTURAS & 0.00 & 0.00 & 0.00 & 0.00 & 0.00 & 0.00 & 0.00 & 0.00 & 0.00 & 0.00 \\
\hline Algodón & 0.00 & 0.00 & 0.00 & 0.00 & 0.00 & 0.00 & 0.00 & 0.00 & 0.00 & 0.00 \\
\hline PAPEL Y CARTÓN & 0.00 & 0.00 & 0.00 & 0.00 & 0.00 & 0.00 & 0.00 & 0.00 & 0.00 & 0.00 \\
\hline Otros Productos & 102.20 & 95.90 & 67.64 & 103.37 & 136.02 & 254.00 & 346.20 & 415.40 & 518.90 & 508.00 \\
\hline TOTAL BIENES & 851.00 & 812.00 & 792.45 & 801.50 & 814.00 & 965.50 & 1220.20 & 1320.90 & 1445.70 & 1532.80 \\
\hline
\end{tabular}

Fuente; Elaboración propia con datos del $\mathrm{BCH}$. 
Anexo No. 3

Exportaciones Hondureñas 1999-2007 (valores corrientes en millones de Dólares US\$)

\begin{tabular}{|c|c|c|c|c|c|c|c|c|c|}
\hline & 1999 & 2000 & 2001 & 2002 & 2003 & 2004 & 2005 & 2006 & 2007 \\
\hline BANANO & 38.10 & 124.20 & 204.70 & 172.30 & 133.30 & 208.80 & 260.30 & 241.30 & 289.30 \\
\hline CAFÉ & 256.10 & 362.50 & 178.90 & 190.20 & 192.00 & 277.20 & 366.30 & 425.80 & 518.30 \\
\hline MADERA & 27.50 & 33.50 & 35.30 & 33.90 & 32.90 & 35.00 & 43.30 & 39.20 & 44.50 \\
\hline Carne refrigerada & 2.30 & 0.00 & 0.00 & 0.00 & 0.00 & 0.00 & 0.00 & 0.00 & 0.00 \\
\hline ACEÍTE DE PALMA & 0.00 & 16.30 & 31.40 & 29.90 & 55.50 & 59.30 & 56.30 & 74.80 & 121.20 \\
\hline PLATA & 5.40 & 2.70 & 3.50 & 2.90 & 2.50 & 5.80 & 5.20 & 11.00 & 17.60 \\
\hline PLOMO & 3.70 & 1.00 & 1.80 & 1.60 & 1.80 & 5.40 & 5.50 & 8.60 & 24.10 \\
\hline ZINC & 50.20 & 18.40 & 12.20 & 14.30 & 15.10 & 20.30 & 19.60 & 60.70 & 58.00 \\
\hline AZÚCAR & 5.30 & 7.80 & 28.10 & 15.60 & 13.20 & 14.90 & 24.80 & 29.60 & 19.40 \\
\hline CAMARÓN CULTIVADO & 127.40 & 90.60 & 93.20 & 78.30 & 117.90 & 128.80 & 124.50 & 156.40 & 120.30 \\
\hline CAMARÓN DE EXTRACCIÓN & 22.40 & 29.40 & 13.60 & 10.40 & 5.00 & 7.60 & 7.90 & 5.50 & 5.60 \\
\hline Camaron & 0.00 & 0.00 & 0.00 & 0.00 & 0.00 & 0.00 & 0.00 & 0.00 & 0.00 \\
\hline Camarones y Langostas & 0.00 & 0.00 & 0.00 & 0.00 & 0.00 & 0.00 & 0.00 & 0.00 & 0.00 \\
\hline LANGOSTAS & 39.50 & 23.40 & 29.90 & 37.00 & 42.00 & 47.20 & 48.20 & 50.10 & 32.00 \\
\hline TILAPIAS & 0.00 & 5.30 & 7.50 & 17.50 & 16.60 & 23.60 & 41.30 & 48.50 & 55.50 \\
\hline MELONES Y SANDÍAS & 47.00 & 34.40 & 25.20 & 28.50 & 35.50 & 35.00 & 35.90 & 35.60 & 46.20 \\
\hline PIÑAS & 19.20 & 11.30 & 9.60 & 8.70 & 16.60 & 21.60 & 20.20 & 18.00 & 21.20 \\
\hline LEGUMBRES Y HORTALIZAS & 0.00 & 16.50 & 17.00 & 30.50 & 35.00 & 33.10 & 36.60 & 42.40 & 44.80 \\
\hline PREPARACIONES LEGUMBRES Y FRUTAS & 0.00 & 20.70 & 17.90 & 19.10 & 22.80 & 20.80 & 25.00 & 30.80 & 37.10 \\
\hline PUROS O CIGARROS & 0.00 & 10.50 & 8.70 & 9.60 & 59.40 & 71.30 & 75.20 & 74.90 & 89.40 \\
\hline CIGARRILLOS & 0.00 & 6.90 & 6.00 & 15.20 & 23.90 & 18.90 & 13.10 & 23.70 & 27.10 \\
\hline TABACO & 10.50 & 12.60 & 11.10 & 20.30 & 11.90 & 8.00 & 12.90 & 15.60 & 16.90 \\
\hline Pure y pasta de Frutas & 0.00 & 0.00 & 0.00 & 0.00 & 0.00 & 0.00 & 0.00 & 0.00 & 0.00 \\
\hline MANUFACTURAS DE MADERA & 0.00 & 32.00 & 28.90 & 25.00 & 38.80 & 38.60 & 40.80 & 32.20 & 40.00 \\
\hline MUEBLES DE MADERA & 0.00 & 12.30 & 6.10 & 5.20 & 28.80 & 24.50 & 27.50 & 27.70 & 26.20 \\
\hline TEXTILES & 0.00 & 7.40 & 5.30 & 5.20 & 7.20 & 17.40 & 56.90 & 42.20 & 51.30 \\
\hline JABONES Y DETERGENTES & 38.70 & 45.70 & 54.80 & 25.80 & 34.20 & 53.40 & 42.50 & 45.90 & 44.00 \\
\hline PLÁSTICOS Y SUS MANUFACTURAS & 0.00 & 7.80 & 11.90 & 12.60 & 20.90 & 49.60 & 58.70 & 78.40 & 134.20 \\
\hline Algodón & 0.00 & 0.00 & 0.00 & 0.00 & 0.00 & 0.00 & 0.00 & 0.00 & 0.00 \\
\hline PAPEL Y CARTÓN & 0.00 & 5.40 & 5.40 & 8.30 & 20.10 & 23.80 & 24.80 & 28.80 & 51.10 \\
\hline Otros Productos & 471.10 & 352.60 & 352.40 & 341.40 & 305.60 & 317.00 & 355.50 & 368.50 & 526.20 \\
\hline TOTAL BIENES & 1164.40 & 1291.20 & 1200.10 & 1159.30 & 1288.40 & 1566.80 & 1829.20 & 2016.30 & 2461.30 \\
\hline
\end{tabular}

Fuente; Elaboración propia con datos del $\mathrm{BCH}$. 
Anexo No. 4

Exportaciones Hondureñas 2007-2015 (valores corrientes en millones de Dólares US\$)

\begin{tabular}{|c|c|c|c|c|c|c|c|c|}
\hline & 2008 & 2009 & 2010 & 2011 & 2012 & 2013 & 2014 & 2015 \\
\hline BANANO & 383.80 & 327.20 & 335.40 & 397.80 & 448.90 & 490.10 & 456.00 & 505.00 \\
\hline CAFÉ & 617.90 & 531.50 & 722.60 & 1358.40 & 1402.40 & 749.80 & 838.50 & 986.00 \\
\hline MADERA & 36.30 & 24.10 & 22.30 & 17.60 & 20.20 & 21.50 & 30.70 & 28.70 \\
\hline Carne refrigerada & 0.00 & 0.00 & 0.00 & 0.00 & 0.00 & 0.00 & 0.00 & 0.00 \\
\hline ACEÍTE DE PALMA & 216.70 & 126.50 & 165.70 & 270.10 & 319.20 & 286.40 & 309.60 & 242.50 \\
\hline PLATA & 21.40 & 17.40 & 25.20 & 40.60 & 23.20 & 25.90 & 32.40 & 16.30 \\
\hline PLOMO & 21.10 & 18.50 & 28.70 & 26.20 & 14.00 & 15.90 & 20.00 & 10.10 \\
\hline ZINC & 26.10 & 25.10 & 42.70 & 39.10 & 26.20 & 28.50 & 27.10 & 21.60 \\
\hline AZÚCAR & 20.90 & 29.10 & 44.40 & 41.10 & 56.30 & 66.60 & 74.30 & 61.10 \\
\hline CAMARÓN CULTIVADO & 98.60 & 113.10 & 135.20 & 157.80 & 176.00 & 225.30 & 243.60 & 177.90 \\
\hline CAMARÓN DE EXTRACCIÓN & 2.40 & 2.30 & 4.50 & 5.60 & 2.00 & 5.70 & 8.40 & 3.50 \\
\hline Camaron & 0.00 & 0.00 & 0.00 & 0.00 & 0.00 & 0.00 & 0.00 & 0.00 \\
\hline Camarones y Langostas & 0.00 & 0.00 & 0.00 & 0.00 & 0.00 & 0.00 & 0.00 & 0.00 \\
\hline LANGOSTAS & 44.10 & 23.00 & 36.60 & 42.40 & 44.30 & 42.20 & 38.10 & 51.80 \\
\hline TILAPIAS & 63.00 & 55.80 & 56.80 & 62.80 & 60.40 & 65.20 & 74.10 & 64.20 \\
\hline MELONES Y SANDÍAS & 44.50 & 49.10 & 42.90 & 54.00 & 51.50 & 58.80 & 64.90 & 56.80 \\
\hline PIÑAS & 21.30 & 23.00 & 18.10 & 20.90 & 20.50 & 19.80 & 20.70 & 25.80 \\
\hline LEGUMBRES Y HORTALIZAS & 52.30 & 51.00 & 47.40 & 72.70 & 57.90 & 63.50 & 71.10 & 73.30 \\
\hline PREPARACIONES LEGUMBRES Y FRUTAS & 27.90 & 28.70 & 30.40 & 34.70 & 40.00 & 41.10 & 69.60 & 62.50 \\
\hline PUROS O CIGARROS & 96.20 & 86.20 & 73.50 & 81.40 & 88.90 & 92.60 & 87.40 & 93.20 \\
\hline CIGARRILLOS & 25.70 & 27.10 & 27.90 & 30.50 & 30.80 & 32.20 & 32.90 & 34.70 \\
\hline TABACO & 15.00 & 11.30 & 15.80 & 16.60 & 18.30 & 18.30 & 16.90 & 21.40 \\
\hline Pure y pasta de Frutas & 0.00 & 0.00 & 0.00 & 0.00 & 0.00 & 0.00 & 0.00 & 0.00 \\
\hline MANUFACTURAS DE MADERA & 28.90 & 21.30 & 20.40 & 16.60 & 19.60 & 24.40 & 24.80 & 25.50 \\
\hline MUEBLES DE MADERA & 16.90 & 6.20 & 3.90 & 7.10 & 4.60 & 5.30 & 8.40 & 8.30 \\
\hline TEXTILES & 76.90 & 34.40 & 44.80 & 57.70 & 78.00 & 86.30 & 87.20 & 73.10 \\
\hline JABONES Y DETERGENTES & 52.30 & 52.30 & 59.60 & 68.90 & 79.70 & 97.00 & 88.10 & 95.70 \\
\hline PLÁSTICOS Y SUS MANUFACTURAS & 83.40 & 46.50 & 52.80 & 78.40 & 96.00 & 90.10 & 88.10 & 86.80 \\
\hline Algodón & 0.00 & 0.00 & 0.00 & 0.00 & 0.00 & 0.00 & 0.00 & 0.00 \\
\hline PAPEL Y CARTÓN & 63.00 & 48.70 & 71.20 & 63.70 & 85.30 & 94.10 & 106.90 & 112.50 \\
\hline Otros Productos & 627.00 & 516.40 & 606.10 & 803.90 & 1031.10 & 1041.60 & 1037.70 & 886.30 \\
\hline TOTAL BIENES & 2783.40 & 2295.80 & 2734.80 & 3866.40 & 4295.40 & 3787.90 & 3957.60 & 3824.50 \\
\hline
\end{tabular}

Fuente: Elaboración propia con datos del $\mathrm{BCH}$. 


\section{Anexo No. 5}

IHH, Crecimiento económico, coeficiente de apertura económica de Honduras y crecimiento de las exportaciones reales 1980-2015

\begin{tabular}{|c|c|c|c|c|}
\hline Año & $\begin{array}{l}\text { Crecimiento } \\
\text { Económico }\end{array}$ & $\begin{array}{l}\text { Crecimiento de } \\
\text { las Exportaciones }\end{array}$ & $\begin{array}{c}\text { Indice Herfindahl } \\
\text { Hirschman }\end{array}$ & $\begin{array}{c}\text { Indice de Apertura } \\
\text { Económica }\end{array}$ \\
\hline 1980 & 0.60 & & 2041.19 & 57.29 \\
\hline 1981 & -1.22 & -9.50 & 1762.47 & 52.84 \\
\hline 1982 & -2.07 & -19.45 & 2022.15 & 42.69 \\
\hline 1983 & 0.91 & -0.51 & 3157.15 & 42.23 \\
\hline 1984 & 6.33 & 3.54 & 1970.25 & 43.19 \\
\hline 1985 & 5.63 & 4.97 & 2253.83 & 41.42 \\
\hline 1986 & 5.33 & 9.31 & 2569.52 & 40.64 \\
\hline 1987 & 6.64 & -5.63 & 2525.13 & 37.48 \\
\hline 1988 & 1.75 & -4.01 & 2540.06 & 35.22 \\
\hline 1989 & 2.73 & 29.68 & 2459.92 & 41.75 \\
\hline 1990 & 2.79 & -46.30 & 2747.29 & 47.39 \\
\hline 1991 & -3.32 & -16.10 & 2091.98 & 53.34 \\
\hline 1992 & 6.07 & 1.16 & 1652.38 & 54.70 \\
\hline 1993 & 6.50 & 1.83 & 1436.13 & 59.84 \\
\hline 1994 & 0.21 & -9.09 & 1558.90 & 71.06 \\
\hline 1995 & 6.19 & -1.11 & 2027.26 & 70.06 \\
\hline 1996 & 1.87 & -1.04 & 2004.69 & 83.35 \\
\hline 1997 & 4.60 & -2.63 & 2133.37 & 84.88 \\
\hline 1998 & 3.59 & 0.37 & 2198.18 & 85.19 \\
\hline 1999 & -0.74 & -12.18 & 2321.87 & 86.40 \\
\hline 2000 & 7.29 & 3.46 & 1729.49 & 85.44 \\
\hline 2001 & 2.72 & -7.99 & 1504.93 & 82.39 \\
\hline 2002 & 3.75 & -4.46 & 1468.21 & 80.66 \\
\hline 2003 & 4.55 & 1.04 & 1087.26 & 84.52 \\
\hline 2004 & 6.23 & 8.08 & 1070.50 & 91.74 \\
\hline 2005 & 6.05 & 6.34 & 1121.30 & 95.19 \\
\hline 2006 & 6.57 & 39.21 & 1082.25 & 115.23 \\
\hline 2007 & 6.19 & 2.80 & 1174.61 & 118.69 \\
\hline 2008 & 4.23 & -0.58 & 1322.98 & 119.96 \\
\hline 2009 & -2.43 & -27.66 & 1356.49 & 83.62 \\
\hline 2010 & 3.73 & 23.99 & 1448.95 & 95.78 \\
\hline 2011 & 3.84 & 18.12 & 1870.03 & 107.86 \\
\hline 2012 & 4.13 & 1.17 & 1854.39 & 106.49 \\
\hline 2013 & 2.79 & -7.89 & 1454.39 & 101.40 \\
\hline 2014 & 3.09 & -2.00 & 1415.78 & 98.08 \\
\hline 2015 & 3.64 & 7.99 & 1486.55 & 107.82 \\
\hline
\end{tabular}

Fuente: Elaboración propia con datos del $\mathrm{BCH}$. 